\title{
Correlations between HER2 Expression and Other Prognostic Factors in Breast Cancer: Inverse Relations with the Ki-67 Index and P53 Status
}

\author{
Mehrdad Payandeh ${ }^{1}$,Ali Shahriari-Ahmadi², Masoud Sadeghi' ${ }^{1 *}$, Edris Sadeghi $^{1}$
}

\begin{abstract}
Background: Overexpression or amplification of human epidermal growth factor receptor-2 (HER2) is associated with grade of malignancy and a poor prognosis in breast cancer (BC). The aim of this study was to evaluate of value of HER2 as a prognostic marker, and to analyze associations with common histopathological parameters in BC cases. Materials and Methods: Between of 2007 to 2014, 260 patients with BC referred to Oncology Clinic provided cancer tissue samples which underwent immunohistochemistry (IHC) for markers. ER and PR positivity was defined as $\geq 10 \%$ positive tumor cells with nuclear staining. HER2-positive was defined as either HER2 gene amplification by fluorescent in situ hybridization (FISH) or scored as 3+ by IHC. For HER2 (2+), FISH was performed to determine HER2 positivity. Results: The mean age at diagnosis for the patients with HER2-negative was significantly higher than in HER2-positive cases. Also, there were significant correlations between histological grade, nuclear grade, lymph node metastasis, tumor size, $E R$ status, $P R$ status, p53 overexpression and Ki-67 index with HER2 expression. HER2-negative lesions were of higher grade and more likely to be ER-negative, PR-negative, p53-positive, lymph node metastasis, with a tumor size $<2 \mathrm{~cm}$ and also Ki-67 $\geq 20 \%$ as compared to the HER2-positive group. Conclusions: Contrary to the results of other studies, HER2-positive tumors in our study had a lower Ki-67 index and were p53-positive. Also, Ki-67 proliferation index $\geq 20 \%$ in more studies was associated with p53-positive.Therefore, tumors which are HER2-positive and have a Ki-67 $\geq 20 \%$ had a more aggressive behavior compared to HER2-positive and Ki-67<20\% lesions.
\end{abstract}

Keywords: Breast cancer - HER2 - Ki-67 - immunohistochemisty - FISH - Western Iran

Asian Pac J Cancer Prev, 17 (3), 1015-1018

\section{Introduction}

Breast cancer $(\mathrm{BC})$ is the most frequent malignancy among women and can be a leading cause of death through middle-aged women that adjuvant chemotherapy, commonly include alkylating agents and anthracyclines, improves survival rate in operable BC (Payandeh et al., 2015a). Recent attention has been directed singularly at molecular classifications of BC (Carey et al., 2006). Human epidermal growth factor receptor-2 (HER2) is a marker of poor prognosis in BC (Ménard et al., 2001). Most studies performed to evaluate the HER2 status were conducted in western countries and compared prognostic value of HER2 in anthracyclines-based to non anthracyclines-based regimens.

In different studies performed in a number of countries, HER2 amplification was found in $20-30 \%$ of breast malignancies (Fountzilas et al.,2012), but in some countries such as Lebanon the higher percentage was reported (el AT et al., 2000). The factors investigated included the presence or absence of lymph node metastasis, nuclear grade, ER/PR status, proliferation (Ki-67), HER2 and p53 overexpression. Immunostaining for ER, PR, p53, Ki-67 and HER2 was carried out as previously described (Kai et al., 2006). Ki-67 is present in all proliferating cells, and there is great interest in its role as a proliferation marker (Nishimura et al., 2010). The proliferation marker Ki-67 is one of the most controversially discussed parameters for treatment decisions in breast cancer patients (Inwald et al., 2013). The prognostic impact of HER2 positivity is reported to be lower in lymph node-negative compared with node-positive women (Ménard et al., 2001). BC aggressiveness can be correlated with proliferation status of tumor cells, which can be ascertained with refernce to tumor grade as well as in terms of the Ki67 index (Haroon et al., 2013).

The purpose of this study was to evaluate of value of HER2 as a prognostic marker, and to analyze the associations between HER 2 and common histopathological parameters in breast cancer. 


\section{Materials and Methods}

\section{Patients}

Between 2007 to 2014, 260 patients with BC referred to Oncology Clinic in Kermanshah city, Iran. We analyzed age, sex, grade, tumor size, vascular invasion, hormone receptors status, p53 overexpression, Ki-67 index in the patients. A sufficient sample size was selected from any patient and the slides were stained by hematoxylin and eosin method. Then 4-micron sections were prepared for staining with $\mathrm{H} \& \mathrm{E}$ for IHC markers (Ki-67, ER, PR, p53 and HER2). ER and PR positivity was defined as $\geq 10 \%$ positive tumor cells with nuclear staining. HER2positive was defined as either HER2 gene amplification by fluorescent in situ hybridization (FISH) or scored as $3+$ by IHC (Ahmadi et al., 2015). For HER2 (2+), FISH was performed to determine HER2 positivity. Age, tumor size, lymph node involvement, histological grade, nuclear grade, vascular invasion, p53 and Ki-67 index were other factors that determined in a lot of patients. Ki-67 index was divided to scores ( $<20 \%$ and $\geq 20 \%$ ).

\section{Statistical analysis}

The correlation between the variables was done by IBM SPSS statistics 19. Chi-square test was used to analyze the significance of correlation between the expression of HER2 and other parameters and $\mathrm{P}<0.05$ was considered significant.

\section{Results}

The Table 1 shows correlation between the number of variables with HER2 expression in NHL patients. The mean age at diagnosis for the patients with HER2negative was higher than HER2-positive and this correlation was significant $(\mathrm{P}=0.012)$. Also, there was significant correlation between histological grade, nuclear grade, lymph node metastasis, tumor size, ER status, PR status, p53 overexpression and Ki-67 index with HER2 expression. The patients with HER2-negative were more with higher grade and also percentage of patients with ER-negative, PR-negative, p53-positive, lymph node metastasis, tumor size $<2 \mathrm{~cm}$ and $\mathrm{Ki}-67 \geq 20 \%$ were more in HER2-negative group vs. HER2-positive group.

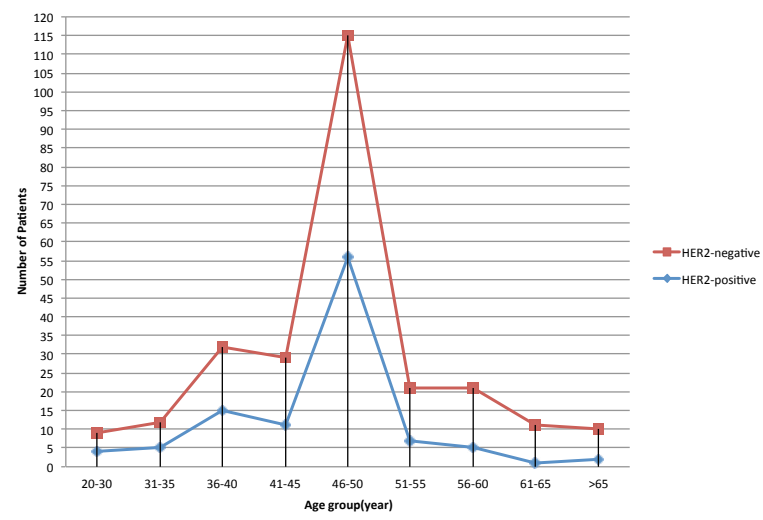

Figure 1. Numbers of patients with Breast Cancer (HER2-positive vs. HER2-negative) Based on Age Groups
We divided HER2-positive patients to two groups based on Ki-67 index (HER2-positive and Ki-67<20\% (group 1) vs. HER2-positive and Ki-67 $\geq 20 \%$ (group 2)) (Table 2). There was just significant correlation between two groups for ER status and p53 overexpression.

Table 1. Correlation between Baseline Characteristics of breast cancer patients and HER2 expression $(n=260)$

\begin{tabular}{cccc}
\hline Variables & HER2-positive ${ }^{€}$ & HER2-Negative & P-value $^{\mp}$ \\
\hline Age, year & & & \\
Mean & 45.9 & 48.8 & 0.012 \\
Range & $24-73$ & $26-82$ & \\
$>50$ & $35(33 \%)$ & $63(40.9 \%)$ & 0.123 \\
$\leq 50$ & $71(67 \%)$ & $91(59.1 \%)$ &
\end{tabular}

Histological grade, $\mathrm{n}=237$

0.000

$\begin{array}{lcc}\text { I } & 15(14.9 \%) & 32(23.5 \%) \\ \text { II } & 77(76.2 \%) & 63(46.3 \%) \\ \text { III } & 9(8.9 \%) & 41(30.1 \%)\end{array}$

Nuclear grade, $\mathrm{n}=152$

$\begin{array}{lcc}\text { I } & 14(21.9 \%) & 21(23.9 \%) \\ \text { II } & 49(76.6 \%) & 41(46.6 \%) \\ \text { III } & 1(1.6 \%) & 26(29.5 \%)\end{array}$

0.000

Lymph node metastasis, $\mathrm{n}=242$

Yes $\quad 74(74.7 \%) \quad 81(56.6 \%)$

No $\quad 25(25.3 \%) \quad 62(43.4 \%)$

Tumor size $(\mathrm{cm}), \mathrm{n}=243$

$\geq 2 \quad 93(91.2 \%) \quad 114(80.9 \%)$

$<2 \quad 9(8.8 \%) \quad 27(19.1 \%)$

Vascular invasion, $\mathrm{n}=206$

$\begin{array}{lll}\text { Yes } & 60(70.6 \%) & 75(62 \%) \\ \text { No } & 25(29.4 \%) & 46(38 \%)\end{array}$

ER

Positive 77(72.6\%) 79(51.3\%)

PR

Negative 29(27.4\%) $\quad 75(48.7 \%)$

0.000

0.006

$78(50.6 \%)$

$76(49.4 \%)$

$\mathrm{P} 53, \mathrm{n}=231$

Positive $\quad 30(32.3 \%) \quad 74(53.6 \%)$

Negative 63(67.7\%) 64(46.4\%)

$\mathrm{Ki}-67$

$\begin{array}{lll}<20 \% & 69(65.1 \%) & 75(48.7 \%) \\ \geq 20 \% & 37(34.9 \%) & 79(51.3 \%)\end{array}$

0.006

All p-values were calculated by Chi-square test, except for mean age was with T-test. ${ }^{€}$ IHC $0,1+$ and $2+$ FISH negative were regarded as negative while IHC $3+$ or $2+$ FISH positive were regarded as positive

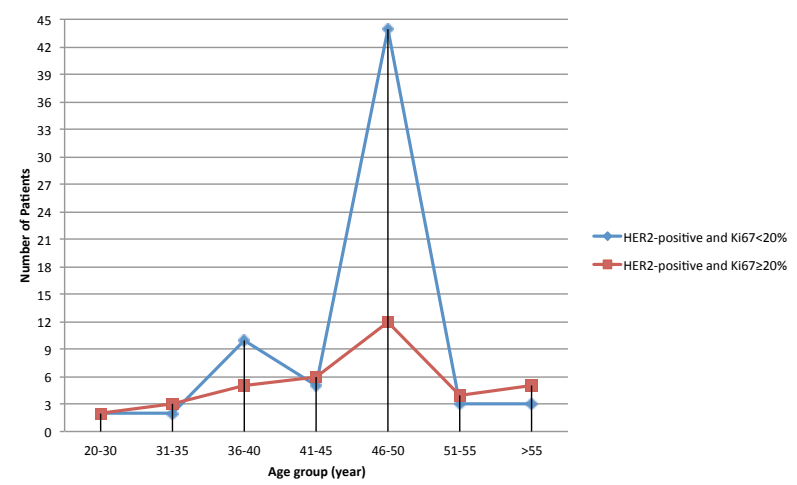

Figure 2. Numbers of Patients with Breast Cancer (HER2-positive and Ki-67<20\% vs. HER2-positive and Ki-67 $\geq 20 \%$ ) Based on Age Groups 
Inverse Correlations between HER2 Expression with the Ki-67 Index and P53 Status in Breast Cancer Cases in Iran

Table 2. Correlations between Baseline Variables of breast cancer Patients with HER2 eExpression and Ki-67 Indices ( $=106)$

\begin{tabular}{|c|c|c|c|}
\hline Variables & Group $1 *$ & Group $2 * *$ & P-value $\neq$ \\
\hline \multicolumn{4}{|l|}{ Age, year } \\
\hline Mean & 45.8 & 46.2 & 0.792 \\
\hline Range & $28-64$ & $24-73$ & \\
\hline$>50$ & $26(37.7 \%)$ & $9(24.3 \%)$ & 0.119 \\
\hline$\leq 50$ & $43(62.3 \%)$ & $28(75.7 \%)$ & \\
\hline \multicolumn{3}{|c|}{ Histological grade, $n=101$} & 0.097 \\
\hline I & $11(16.7 \%)$ & $4(11.4 \%)$ & \\
\hline II & $52(78.8 \%)$ & $25(71.4 \%)$ & \\
\hline III & $3(4.5 \%)$ & $6(17.1 \%)$ & \\
\hline \multicolumn{3}{|c|}{ Lymph node metastasis, $\mathrm{n}=99$} & 0.346 \\
\hline Yes & $48(72.7 \%)$ & $26(78.8 \%)$ & \\
\hline No & $18(27.3 \%)$ & $7(21.2 \%)$ & \\
\hline \multicolumn{3}{|c|}{ Tumor size $(\mathrm{cm}), \mathrm{n}=102$} & 0.394 \\
\hline$\geq 2$ & $7(10.1 \%)$ & $2(6.1 \%)$ & \\
\hline$<2$ & $62(89.9 \%)$ & $31(93.9 \%)$ & \\
\hline \multicolumn{3}{|c|}{ Vascular invasion, $\mathrm{n}=85$} & 0.56 \\
\hline Yes & $39(70.9 \%)$ & $21(70 \%)$ & \\
\hline No & $16(29.1 \%)$ & $9(30 \%)$ & \\
\hline \multicolumn{3}{|l|}{ ER } & 0.392 \\
\hline Positive & $49(71 \%)$ & $28(75.7 \%)$ & \\
\hline Negative & $20(29 \%)$ & $9(24.3 \%)$ & \\
\hline \multicolumn{3}{|l|}{ PR } & 0.019 \\
\hline Positive & $41(59.4 \%)$ & $30(81.1 \%)$ & \\
\hline Negative & $28(40.6 \%)$ & $7(18.9 \%)$ & \\
\hline \multicolumn{3}{|c|}{$\mathrm{P} 53, \mathrm{n}=93$} & 0.001 \\
\hline Positive & $11(19 \%)$ & $19(54.3 \%)$ & \\
\hline Negative & $47(81 \%)$ & $16(45.7 \%)$ & \\
\hline
\end{tabular}

$\neq$ All p-values were calculated by Chi-square test, except for mean age was with T-test; * Group 1: HER2-positive and Ki-67<20\%, **Group 2: HER2-positive and $\mathrm{Ki}-67 \geq 20 \%$; $€$ IHC $0,1+$ and $2+$ FISH negative were regarded as negative while IHC $3+$ or $2+$ FISH positive were regarded as positive.

Therefore, PR-negative or p53-positive was more in group 1 compared with group 2.

Figure 1 and Figure 2 show the prevalence of patients with $\mathrm{BC}$ based on age group. The most percentage of patients is located in $46-50$ years.

\section{Discussion}

Invasive breast carcinoma is the most common malignant tumor in women worldwide that trastuzumab therapy (Herceptin) use for breast tumors with HER2 overexpression (Payandeh et al., 2015b). HER2 belongs to a family of four transmembrane receptors involved in signal transduction pathways that regulate cell growth and differentiation. Overexpression or amplification of HER2 is associated with malignancy and a poor prognosis in BC (Yarden Y,2001). HER2-positive BCs tended to be larger and were less likely to express estrogen receptors, and the incidence rate was higher in patients less than 40 years old (Swede et al., 2001). In our study, HER2-positive BCs occurred more in 46 to 50 years. A retrospective study assessed HER2-positive patients who were diagnosed with brain metastases. The median age of the 432 patients was 54 years (range, 20-86 years) (Hayashi et al., 2015). Our study showed that in patients with HER2-positive or HER2-negative, the mean age was higher than 45 years. Camerini et al.(2011) reported that HER2 expression had not correlation with age, ER expression, PR expression, Ki-67 and just had with grade. A research (Shokouh et al., 2015), showed that significant correlation was observed between tumor grade with HER2 overexpression. Higher grades had significantly greater positivity in Ki-67 index and HER2-positive tumors had a higher Ki-67 index. Moreover, in young patients with breast carcinoma tumors, the rates of $\mathrm{Ki}-67$ with the overexpression of HER 2 and p53 mutations are higher, and it shows a more aggressive behavior than other tumors (Shokouh et al., 2015). The Ki-67 expression was associated significantly with histological grade, ER, PR, HER2, and p53 status (Li et al., 2014). A higher Ki-67 index $(\geq 20 \%)$ significantly correlated with a higher grade of malignancy, such as negative ER/PR, higher grade, p53 overexpression and HER2- positive (Nishimura et al., 2010). Wiesner et al. (2009) reported that a Ki-67 proliferation index $\geq 20 \%$ was found to be associated with all prognostic factors such ER, PR, HER2 and nuclear grade. High p53 expression was positively correlated with HER 2 score and Ki-67 expression (Yamamoto et al., 2014). HER 2 positivity was detected more significantly in patients with p53 overexpression (Lee et al., 2011). There was no significant correlation between HER-2 and age, tumor size, lymph node status, ER, and PR. There was significant correlation between HER-2 with tumor grade and p53 (Patnayak et al., 2015). In our study, there was significant correlation between histological grade, nuclear grade, lymph node metastasis, tumor size, ER status, PR status, p53 overexpression and Ki-67 index with HER2 expression. HER2 inversely correlated with higher grade, higher Ki-67 index ( $\geq 20 \%)$ and p53 positively and also directly with ER expression, PR expression, lymph node metastasis, lower tumor size. Also, in HER2-positive, patients with $\mathrm{Ki}-67 \geq 20 \%$ had significantly higher ER expression and p53-positive compared to HER2-positive and $\mathrm{Ki}-67<20 \%$ had higher p53-positive.

In conclusions, Contrary to the results of other studies, HER2-positive tumors in our study had a lower Ki-67 index and p53-positive. Also, Ki-67 proliferation index $\geq 20 \%$ in more studies associated with p53-positive. Therefore, tumors with HER2-positive and Ki- $67 \geq 20 \%$ had a more aggressive behavior compared to HER2positive and $\mathrm{Ki}-67<20 \%$.

\section{References}

Ahmadi AS, Mahdipour L, Payandeh M, et al (2015). Epidemiology, pathology and histochemistry features in women with breast cancer. Am J Cancer Prev, 3, 54-7.

Camerini A, Donati S, Viacava P, et al (2011). Evaluation of HER2 and p53 expression in predicting response to docetaxel-based first-line chemotherapy in advanced breast cancer. J Exp Clin Cancer Res, 30, 38.

Carey LA, Perou CM, Livasy CA, et al (2006). Race, breast cancer subtypes, and survival in the Carolina Breast Cancer Study. JAMA, 295, 2492-502.

el AT, Khalifa A, Kamel AS (2000). Immunohistochemical expression of $\mathrm{p} 53$ and c-erbB2 proteins in breast cancer in Egypt. Anticancer Res, 20, 2145-50.

Fountzilas G, Valavanis C, Kotoula V, et al (2012). HER2 and 
TOP2A in high-risk early breast cancer patients treated with adjuvant epirubicin-based dose-dense sequential chemotherapy. J Transl Med, 10, 10.

Haroon S, Hashmi AA, Khurshid A, et al (2013). Ki67 index in breast cancer: correlation with other prognostic markers and potential in Pakistani patients. Asian Pac J Cancer Prev, 14, 4353-8.

Hayashi N, Niikura N, Masuda N, et al (2015). Prognostic factors of HER2-positive breast cancer patients who develop brain metastasis: a multicenter retrospective analysis. Breast Cancer Res Treat, 149, 277-84.

Inwald EC, Klinkhammer-Schalke M, Hofstädter F, et al (2013). $\mathrm{Ki}-67$ is a prognostic parameter in breast cancer patients: results of a large population-based cohort of a cancer registry. Breast Cancer Res Treat, 139, 539-52.

Kai K, Nishimura R, Arima N, et al (2006). p53 expression status is a significant molecular marker in predicting the time to endocrine therapy failure in recurrent breast cancer: a cohort study. Int J Clin Oncol, 11, 426-33.

Lee DS, Kim SH, Suh YJ, et al (2011). Clinical implication of p53 overexpression in breast cancer patients younger than 50 years with a triple-negative subtype who undergo a modified radical mastectomy. Jpn J Clin Oncol, 41, 854-66.

Li FY, Wu SG, Zhou J, et al (2014). Prognostic value of Ki-67 in breast cancer patients with positive axillary lymph nodes: a retrospective cohort study. PLoS One, 9, 87264.

Ménard S, Fortis S, Castiglioni F, et al (2001). HER2 as a prognostic factor in breast cancer. Oncol, 61, 67-72.

Nishimura R, Osako T, Okumura Y, et al (2010). Ki-67 as a prognostic marker according to breast cancer subtype and a predictor of recurrence time in primary breast cancer. Exp Ther Med, 1, 747-54.

Patnayak R, Jena A, Rukmangadha N, et al (2015). Hormone receptor status (estrogen receptor, progesterone receptor), human epidermal growth factor-2 and p53 in South Indian breast cancer patients: A tertiary care center experience. Indian J Med Paediatr Oncol, 36, 117-22.

Payandeh M, Khodarahmi R, Sadeghi M, et al (2015a). Appearance of acute myelogenous leukemia (AML) in a patient with breast cancer after adjuvant chemotherapy: case report and review of the literature. Iran J Cancer Prev, 8, 125-8.

Payandeh M, Sadeghi M, Sadeghi E, et al (2015b). Clinicopathology figures and long-term effects of tamoxifen plus radiation on survival of women with invasive ductal carcinoma and triple negative breast cancer. Asian Pac J Cancer Prev, 16, 4863-7.

Shokouh TZ, Ezatollah A, Barand P (2015). Interrelationships Between Ki67, HER2/neu, p53, ER, and PR status and their associations with tumor grade and lymph node involvement in breast carcinoma subtypes: retrospective-observational analytical study. Med (Baltimore), 94, 1359.

Swede H, Moysich KB, Freudenheim JL, et al (2001). Breast cancer risk factors and HER2 over-expression in tumors. Cancer Detect Prev, 25, 511-9.

Wiesner FG, Magener A, Fasching PA, (2009). Ki-67 as a prognostic molecular marker in routine clinical use in breast cancer patients. Breast, 18, 135-41.

Yamamoto M, Hosoda M, Nakano K, et al (2014). p53 accumulation is a strong predictor of recurrence in estrogen receptor-positive breast cancer patients treated with aromatase inhibitors. Cancer $S c i, \mathbf{1 0 5}, 81-8$.

Yarden Y (2001). Biology of HER2 and its importance in breast cancer. Oncol, 61, 1-13. 